
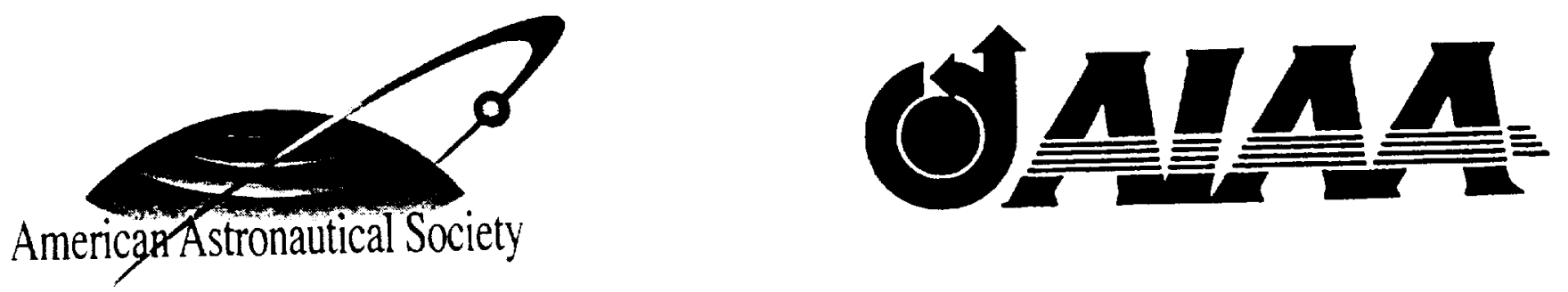

\title{
The Feasibility of a Galileo-Style Tour of the Uranian Satellites
}

Andrew F. Heaton and James M. Longuski

School of Aeronautics and Astronautics Purdue University

West Lafayette, Indiana 47907-1282

\section{AAS/AIAA Astrodynamics Specialists Conference}

Quebec City, Quebec, Canada July 30-August 2, 2001

AAS Publications Office, P.O. Box 28130, San Diego, CA 92198 


\title{
THE FEASIBILITY OF A GALILEO-STYLE TOUR OF THE URANIAN SATELLITES ${ }^{*}$
}

\author{
Andrew F. Heaton ${ }^{\dagger}$ and James M. Longuski ${ }^{\ddagger}$
}

\begin{abstract}
Gravity-assist trajectories have been a key to outer Solar System exploration. In particular, the gravity-assist (1)ur of the Jovian satellites has contributed significantly to the success of the Galileo mission. A comparison of the Jovian system to the Uranian system reveals that the two possess similar satellite/planet mass ratios. Tisserand graphs of the Uranian system also indicate the potential for cours at Uranus. In this paper, we devise tour strategies and design a prototypical tour of the Uranian satellites, proving that tours at Uranus are teasible.
\end{abstract}

\section{INTRODLCTION}

Uranus has a satellite system that is comparable to that of Jupiter. The mass ratios of the Uranian satellites to Lranus are similar to those of the Jovian satellites to Jupiter: which suggests that a tour is feasible (see Table 1). The semimajor axes of the satellites are also similar when scaled to the central planet's radius as shown in Table 2. Yet another similar feature is that the two outermost satellites at Uranus (Titania and Oberon), are most massive, and the two outermost the satellites at Jupiter (Callisto and Ganymede) are also the most massive. In fact, there is a correlation between the mass ratios of the Jovian and Uranian satellites and their semimajor axis, with the exception of Ariel-Io. Starting with the respective innermost satellites, and moving outwards, Ariel's mass ratio is $33 \%$ of lo's. Umbriel's is $54 \%$ of Europa's, Titania's is $53 \%$ of Ganymede's, and Oberon's is $61 \%$ of Callisto's (so the satellite mass ratios of each system are roughly correlated to their semimajor axes). In summary, the Uranian satellite system is almost a smaller replica of the Jovian system in terms of satellite mass ratios and relative size of the semimajor axes. Tables 1 and 2 demonstrate the feasibility of a Galileo-like tour at Uranus.

\footnotetext{
* Copyright $202(x) 1$ by Andrew F. Heaton and Janes M. Longuski. Published by the Americian Astronautical Society with permissten.

Graduate Siudent. School of Aeronauties and Astronautics. Purdue Liniversity. West Latayette. Indiana $47907-1282$. Curtendly Acrospace Engineer. Mission Planning Group. NASA Marshall Space Flight Center. Huntsville. AL, 35812 Huntsville, Alabama

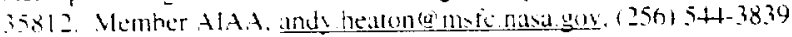

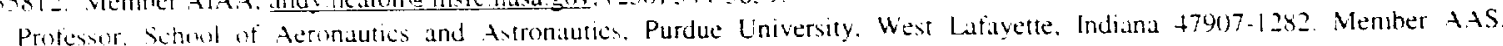

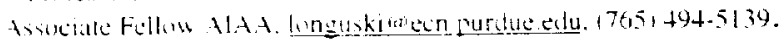


Table 1

MASS RATIO COMPARISON

$\begin{array}{ccc}\text { Satellite } & \begin{array}{c}\frac{\mu}{3} \\ \left(\mathrm{~km}^{2} / \mathrm{s}^{2}\right)\end{array} & \text { Mass Ratio } \\ \frac{\text { Uranian }}{\text { Ariel }} & 98.5 & 1.56 \times 10^{-5} \\ \text { Umbriel } & 78.3 & 1.35 \times 10^{-5} \\ \text { Titania } & 235 & 4.06 \times 10^{-5} \\ \text { Oberon } & 201 & 3.47 \times 10^{-5} \\ & & \\ \text { Jovian } & & \\ \text { Io } & 5934 & 4.68 \times 10^{-5} \\ \text { Europa } & 3196 & 2.52 \times 10^{-5} \\ \text { Ganymede } & 9885 & 7.80 \times 10^{-5} \\ \text { Callisto } & 7172 & 5.66 \times 10^{-5}\end{array}$

Table 2

\section{SEMI-MAJOR AXIS COMPARISON}

\begin{tabular}{cc} 
Satellite & SemimajorAxis \\
Uranian & $\left(\mathrm{R}_{\mathrm{U}}\right)$ \\
\hline Ariel & 7.45 \\
Umbriel & 10.58 \\
Titania & 17.38 \\
Oberon & 23.24 \\
& \\
Jovian & $\left(\mathrm{R}_{\mathrm{J}}\right)$ \\
Io & 5.91 \\
Europa & 9.39 \\
Ganymede & 14.98 \\
Callisto & 26.35
\end{tabular}

\section{TISSERAND GRAPH ANALYSIS}

The Tisserand graph has been developed at Purdue University to faciiitate tour design. ${ }^{1.4}$ This method assumes circular, coplanar orbits for the satellites. Under these assumptions, the intersection of a given spacecraft orbit with a satellite orbit produces a fixed $V_{\infty}$ at any point in the satellite's orbit. Thus, the $V_{\infty}$ of the spacecraft orbit relative to any satellite is a function of the spacecraft orbit periapsis and period. A more detailed explanation can be found in Heaton. et al. ${ }^{2}$ or Strange and Longuski. ${ }^{3}$ This method has been used with great success to design Europa Orbiter tours, and is similar to a method developed by Labunsky, et al. ${ }^{5}$ The method takes its name from Tisserand, who in 1889 


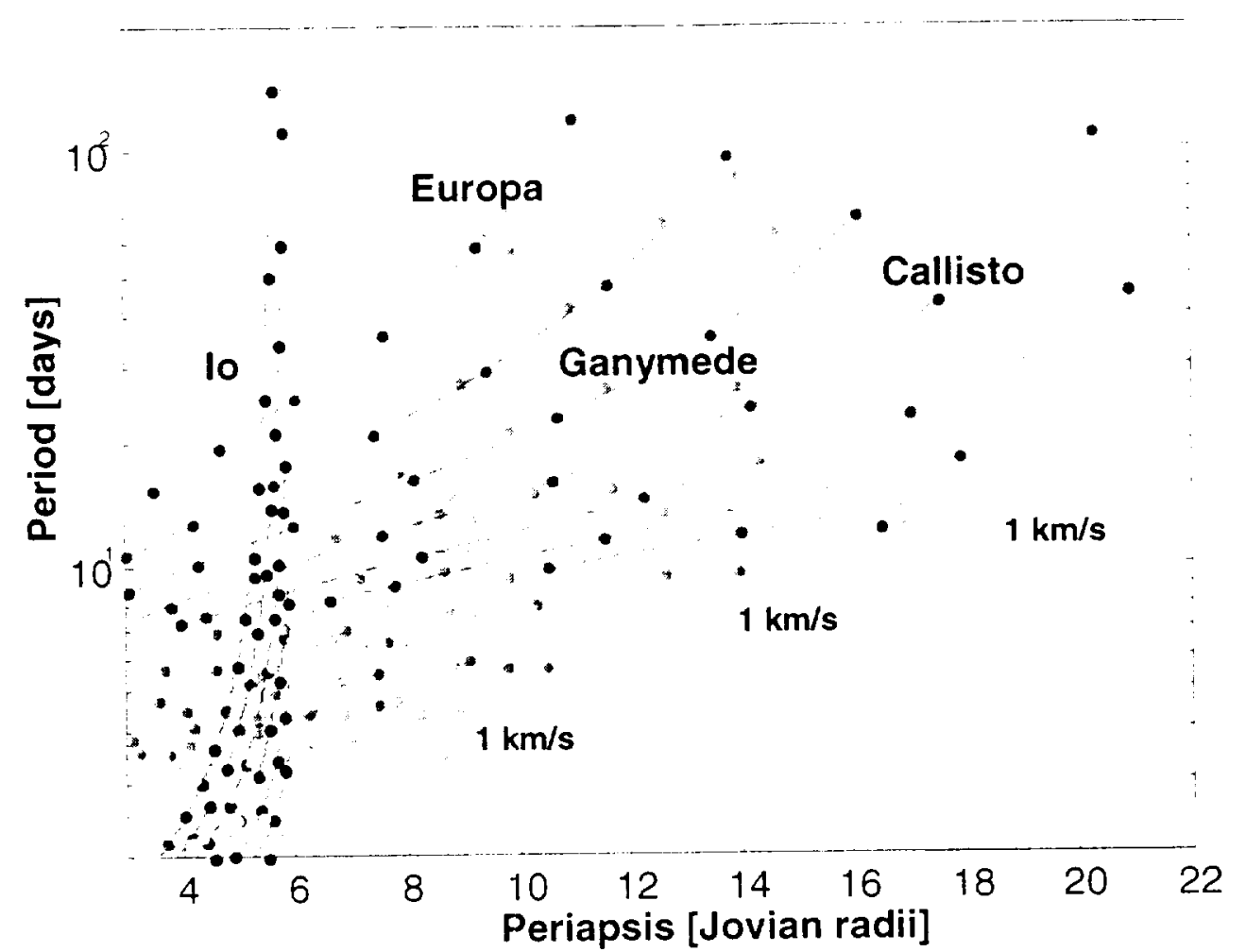

Figure 1 Jovian Tisserand graph. The $V_{\infty}$ contours are in increments of 1 $\mathrm{km} / \mathrm{s}$ in a range from $1 \mathrm{~km} / \mathrm{s}$ to $8 \mathrm{~km} / \mathrm{s}$. Each tick mark represents separate flybys of $100 \mathrm{~km}$.

used a similar relationship (Tisserand's criterion) to explain perturbations of comets by Jupiter. ${ }^{6}$

Tisserand graphs for Jupiter and Uranus are shown in Figures 1 and 2, respectively. We see immediately that these two graphs strongly resemble each other. Some differences also exist, however. First and foremost, the tick marks in the Uranian plot (Figure 2) are closer together. (which agrees well with the mass ratio data presented in Table 1 , since closer tick marks reflect the smaller masses of the Uranian system). The slopes of the $\mathrm{V}_{\infty}$ contours of Oberon are slightly higher than those of Callisto. The same is true of Titania as compared to Ganymede, because the Uranian satellites are relatively farther from the central body. One common factor between the two systems is that the two outermost satellites appear to be the most effective for gravity assists. All the Lranian satellites have less potential for gravity-assist flybys. but the difference in potential is more marked for Umbriel and Ariel (when compared to Europa and Io, respectively) than it is for Titania and Oberon (when compared to Ganymede and Callisto, respectively). Another difference is that the range of satellite-relative $V_{\infty} s$ at Uranus is smaller. This is because Uranus has less gravity and also implies that insertion into Uranian orbit is costlier than insertion into Jovian orbit. 


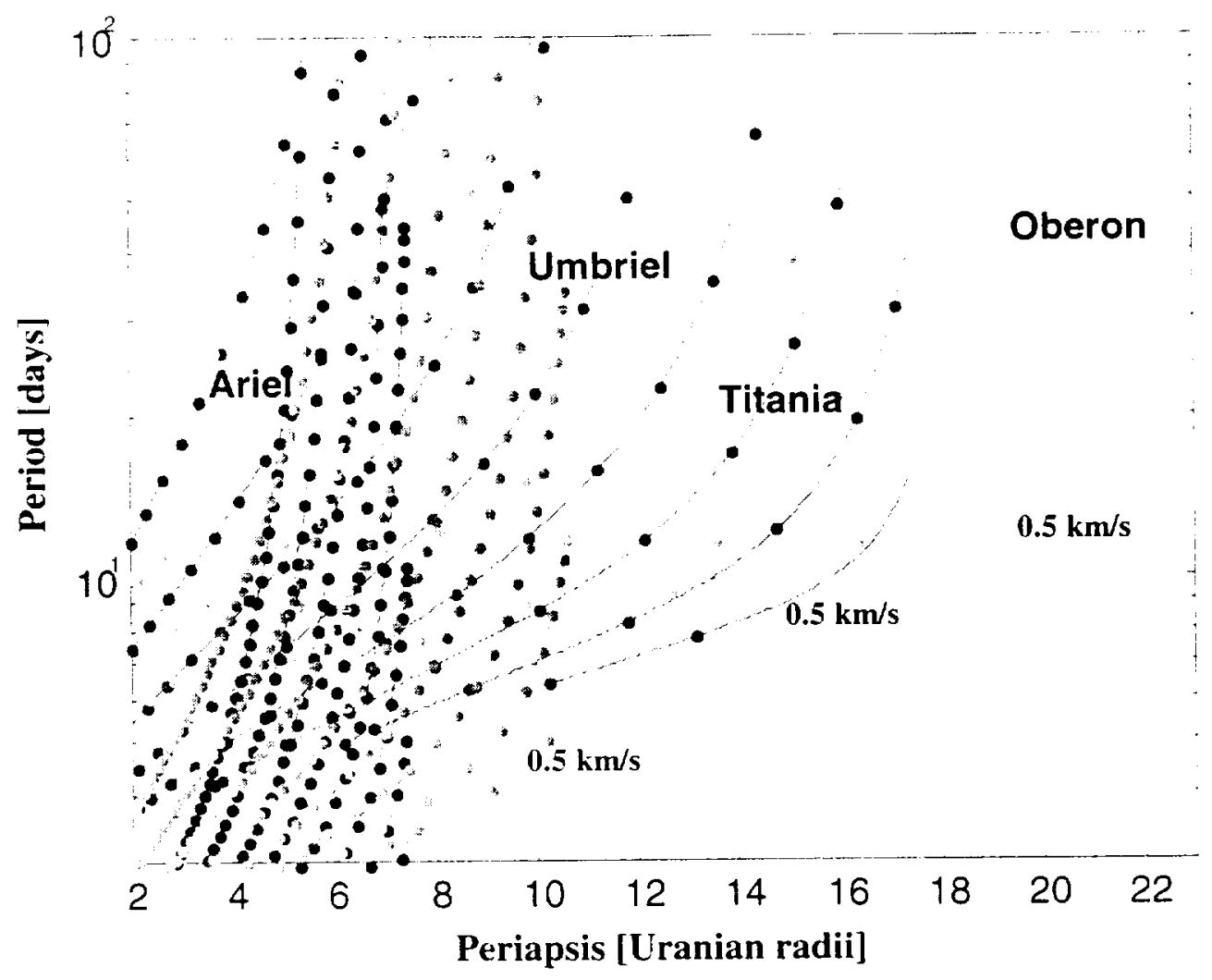

Figure 2 Uranian Tisserand graph. The $V_{\infty}$ contours are in increments of $0.5 \mathrm{~km} / \mathrm{s}$ in a range from $0.5 \mathrm{~km} / \mathrm{s}$ to $4 \mathrm{~km} / \mathrm{s}$. Each tick mark represents separates flybys of $50 \mathrm{~km}$.

\section{ARRIVAL GEOMETRY AND INITIAL CONDITIONS}

Uranus has an obliquity of 97 degrees and its satellites orbit close to the equatorial plane. Thus, any spacecraft arriving there is likely to have a high-inclination initial condition with respect to the satellites. Figure 3 illustrates the arrival geometry problem in more detail for a Hohmann transfer to Uranus from Earth. Note that for certain arrival dates, the arrival $V_{\infty}$ vector coincides with a "zero-inclination insertion", thereby resulting in great savings in $\Delta \mathrm{V}$ (i.e., no propellant cost to place the spacecraft into the satellite plane). However. the Hohmann transfer is not a viable option for a mission to Uranus, since it has a time of flight (TOF) of 16 years. A faster trajectory is highly desirable. A faster trajectory to Uranus, however, implies a different arrival $V_{\infty}$ vector, thereby changing the arrival conditions depicted in Figure 3, and creating the possibility of arriving on different dates with a low inclination. 


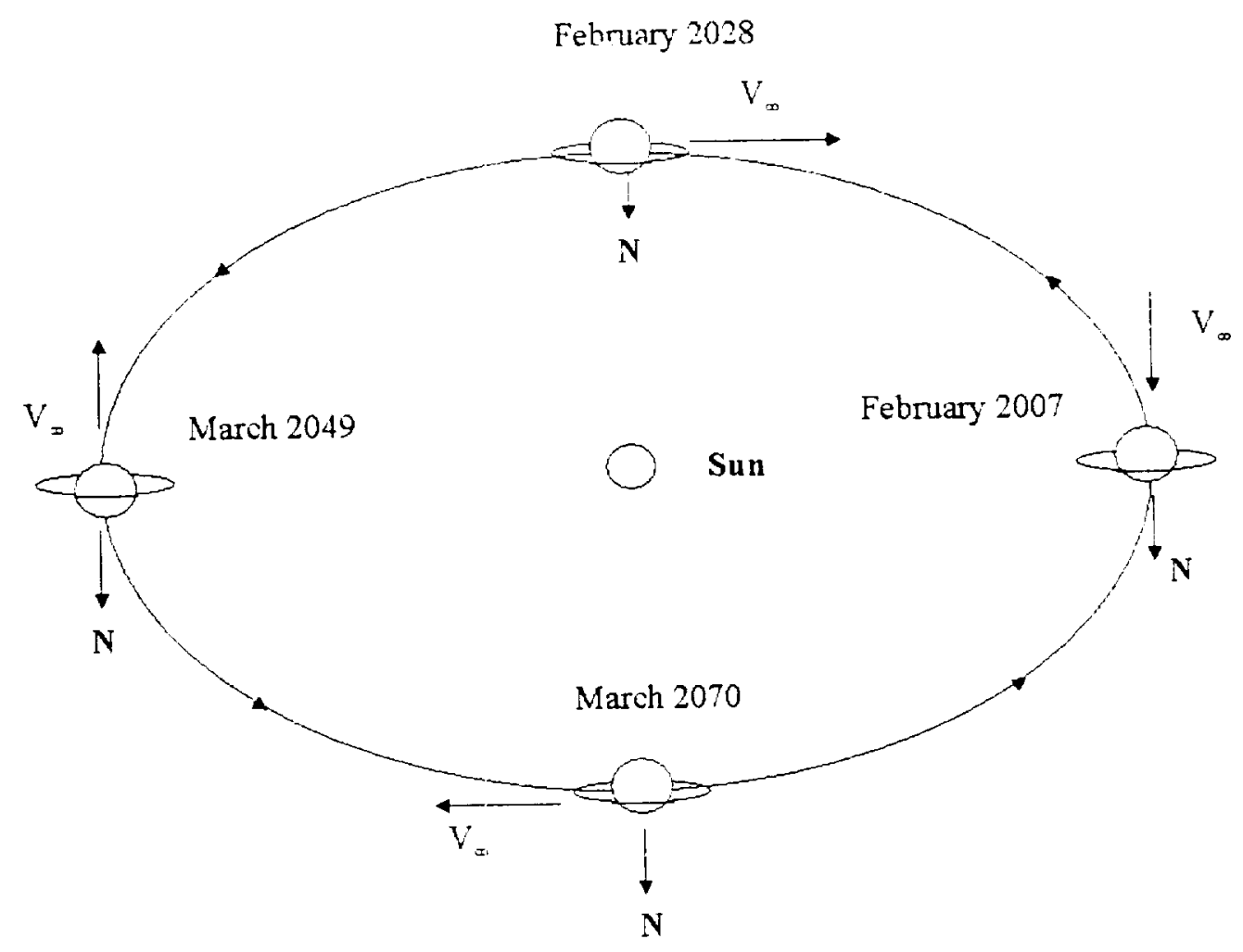

Figure 3 Uranian pole vector migration. Arrival $V_{\infty} s$ are for Hohmann transfers. Arrivals in 2007 and 2049 result in a highly inclined spacecraft orbit. Arrivals in 2028 and 2070 allow orbit insertion into the satellite plane with little or no $\Delta V$.

The rather complex relationship between launch date, arrival date, arrival $\mathrm{V}_{\infty}$ vector, arrival geometry, and launch energy has been explored in detail.' Given current constraints on launch energy and upcoming launch windows, a Jupiter Gravity Assist (JGA) to Jupiter appears to be the best option at the present. For a JGA, a typical arrival $\mathrm{V}_{\infty}$ is about $7.4 \mathrm{~km} / \mathrm{s}$.

Table 3

\section{TYPICAL INITIAL CONDITION FOR URANIAN TOUR ${ }^{\text {a.b }}$}

$\begin{array}{ccc}\frac{V_{\infty}}{(\mathrm{km} / \mathrm{s})} & \frac{\text { Declination }}{(\mathrm{deg})} & \frac{\text { Right Ascension }}{(\mathrm{deg})} \\ 3.27 & 45.1 & 26.3\end{array}$

${ }^{3}$ Coordinate system is JPL's vector standard $(081)$.

${ }^{b}$ Arrives on 5/02/2019, at 14:47:59 GMT.

For the purposes of designing Uranian tours, initial conditions are derived from a JGA trajectory generated using STOUR ${ }^{7-11}$ (the Satellite Tour Design Program). (The 
program STOUR is an automated design tool that tinds gravity-assist tours for satellites systems and for the Solar System). The initial :onditions are used here as the beginning point of Uranian tours. A typical initial condition appears in Table 3.

\section{GUIDELINES AND CONSTRAINTS FOR URANIAN TOUR DESIGN}

Many variables can affect the success of a tour design. For instance, flyby altitude is often limited due to navigational concerns. In order to design a tour, limits must be set on the tour design parameters. In this section, we derive guidelines and constraints for a Uranian tour, and contrast some of them with the guidelines and constraints for the Europa Orbiter tour. ${ }^{2}$

The first issue to be addressed is the purpose of the tour. Is it a general tour of the system similar to that of the Galileo spacecraft, or does it serve a specific purpose as in the case of Europa Orbiter? We elect to use the techniques first developed in the Galileo mission, but in addition we adopt the highly focused goal of the Europa Orbiter mission of reducing $\mathrm{V}_{\infty}$ at a destination satellite. Thus, the tour design in this paper will be similar to the Europa Orbiter tour. The reason for selecting a Europa Orbiter-style tour over a Galileo-style tour is because the Europa-style tour is more challenging, and we wish to rigorously test the feasibility of designing a tour at Uranus. We choose as the goal for the Uranian Orbiter the reduction of the $V_{\infty}$ of arrival at Ariel to less than $1 \mathrm{~km} / \mathrm{s}$. If we can demonstrate the existence of such a tour, then many other tours with less stringent requirements can be designed.

Now that the tour has an objective, guidelines and constraints can be selected. The limit on the periapsis of any orbit in the tour will be $4 R_{L}$. based on the maximum radius of the Uranian rings (about $3+\mathrm{R}_{\mathrm{L}}$ ). The inclination of the initial orbit at Uranus should be less than 20 degrees. based on a trade study which shows that approximately one year of orbit cranking (a flyby that changes only inclination) is required to bring a 20-degree inclined orbit into the equatorial plane (where the satellites reside). ' Larger inclinations require too much time in the orbit-cranking phase. We estimate that a Uranian tour will take about two years, twice as long as a typical Europa Orbiter tour. We require each orbit of the Uranian tour to pass through apoapsis, to allow sufficient time for trajectorycorrection maneuvers between flybys.

The Lranian satellite system is a scaled-down version of the Jovian system. This fact implies that non-targeted encounters occur more frequently at Uranus. since the satellites are closer to each other. Experience has shown that this is, indeed, the case. So the question arises, what is an acceptable tlyby distance for a non-targeted encounter at Uranus? To answer this question, we consider the well-known equation for the maximum deflection angle possible for a given flyby:

$$
\sin \left(\frac{\delta}{2}\right)=\frac{\mu}{\mu+V_{\infty}^{\prime} R_{p}}
$$


In Eq. (1). $\delta$ is the deflection angle, $\mu$ is the gravitational constant of the flyhy body, and $R_{P}$ is the flyby distance from the center of the satellite. Note that the only parameter that must change when we consider Uranus instead of Jupiter is the gravitational constant of the flyby satellite. The $\mathrm{V}_{\infty}$ can be set to the same value for each satellite. The acceptable tlyby distance in the Jovian system is $50,000 \mathrm{~km}$; we are interested in solving for the acceptable distance at Uranus using Eq. (1) for the same $\mathrm{V}_{\infty}$ and deflection angle. Ganymede and Titania are the selected satellites for this assessment, because they are the most massive satellites of Jupiter and Uranus, respectively. We note, however that the acceptable distance is not $50,000 \mathrm{~km}$ but $50,000 \mathrm{~km}$ plus the radius of Ganymede (or $52634 \mathrm{~km}$ ), because Eq. (1) requires the distance from the center of Ganymede to the spacecraft. Thus we get:

$$
\frac{\mu_{T}}{\mu_{T}+V_{\infty}^{2} R_{P T}}=\frac{\mu_{G}}{\mu_{G}+V_{\infty}^{2} R_{P G}}
$$

where the $G$ and $T$ subscripts refer to Ganymede and Titania. respectively. Eq. (2) can be re-arranged to:

$$
\frac{\mu_{\mathrm{T}}}{\mu_{\mathrm{G}}}=\frac{\mu_{\mathrm{T}}+\mathrm{V}_{\infty}^{2} \mathrm{R}_{\mathrm{PT}}}{\mu_{\mathrm{G}}+\mathrm{V}_{\infty}^{2} \mathrm{R}_{\mathrm{PG}}}
$$

Eq. (3) can be further simplified to:

$$
1+\frac{V_{\infty}^{2} R_{P r}}{\mu_{T}}=1+\frac{V_{\infty}^{2} R_{P G}}{\mu_{G}}
$$

Finally, recalling that the $\mathrm{V}_{\infty} \mathrm{s}$ are the same, we get:

$$
\frac{\mathrm{R}_{\mathrm{P}}}{\mathrm{R}_{\mathrm{PG}}}=\frac{\mu_{\mathrm{T}}}{\mu_{\mathrm{G}}}
$$

Equation (5) indicates that the flyby distance for a given amount of bending (and the same $\mathrm{V}_{\infty}$ ) is a function of only the mass ratios of the bodies being compared. The ratio of Ganymede's mass to that of Titania is roughly 42:1, so the equivalent flyby distance at Titania for the same amount of bending is $1188 \mathrm{~km}$. At this point, one might conclude that non-targeted flybys of the Uranian satellites can almost be ignored. However, having the same deflection angle at Titania and Ganymede does not mean that the effect on the respective orbits is the same. In terms of the typical velocity vector diagram for gravity assists, both the satellite and spacecraft velocity vectors will be shorter at Titania than at Ganymede (because the lower gravity of Uranus implies slower velocities). Hence. the same bending at L'ranus has a greater effect on the spacecraft's orbit as the $\mathrm{V}_{\infty}$ vector is rotated through the same angle. Further calculations show that $10,000 \mathrm{~km}$ is sufficient to limit the effects of non-targeted (NT) flybys at Uranus. Hence, we constrain 
NT tlybys to be greater than $25,000 \mathrm{~km}$, which roughly corresponds to the soft limit of $100,000 \mathrm{~km}$ for non-targeted flybys in the Jovian systern."

\section{Table 4}

\section{GUIDELINES AND CONSTRAINTS SUMMARY FOR AN ARIEL ORBITER}

$\begin{array}{cc}\text { Constraint/Guideline } & \underline{\text { Value }} \\ \text { Arrival } \mathrm{V}_{\infty} & <1 \mathrm{~km} / \mathrm{s} \\ \text { Periapsis constraint } & >4 \mathrm{R}_{\mathrm{U}} \\ \text { Initial inclination } & <20 \mathrm{deg} \\ \text { Non-targeted flybys } & >25,000 \mathrm{~km} \\ \text { Flyby altitude } & >50 \mathrm{~km} \\ \text { Time of Flight } & <2.5 \text { years }\end{array}$

The final constraint that must be decided is the flyby altitude. Since the satellites of Uranus are much less massive than those of Jupiter, closer flybys are in general required at Uranus. For this reason, the llyby altitude is set to $50 \mathrm{~km}$ for the Uranian tour. This constraint certainly pushes the limits of what is navigationally feasible. However, for our prototype tour the impact of raising the altitude limit back to back to $100 \mathrm{~km}$ is not that great, since we primarily wish to demonstrate feasibility. The effect of raising the altitude limit is that a few more flybys would be required over the course of the tour. which would lengthen the time of flight. Furthermore, it is not unreasonable to assume that in the future navigational techniques will improve so that the flyby altitude constraint can be lowered. Table 4 presents a summary of all the guidelines and constraints derived in this section.

\section{URANIAN TOUR EXAMPLE}

This section presents a sample tour design at Uranus to demonstrate the feasibility of the concept. The tour design involves three phases: the initial phase, the middle phase and the end phase.

The initial phase addresses the problem of high initial inclination discussed earlier. The solution to this problem is to have many resonant flybys of the first satellite encountered, using crank to reduce the inclination of the orbit. Trade studies performed for Ariel and Titania determined that Titania is more effective for orbit cranking, requiring fewer flybys and less time. This might be expected, due to Titania's greater mass and similarity to Ganymede, which is the best Jovian satellite for reducing inclination. However, Ariel seems to offer better energy reduction from the "steepness" of its curves on the Uranian Tisserand graph. Unfortunately, this potential advantage proves to be insufficient justification for the longer TOF and greater number of flybys necessury if we use Ariel for the initial crank-down. Thus, we choose Titania as the initial flyby body for our tour design at Uranus. 


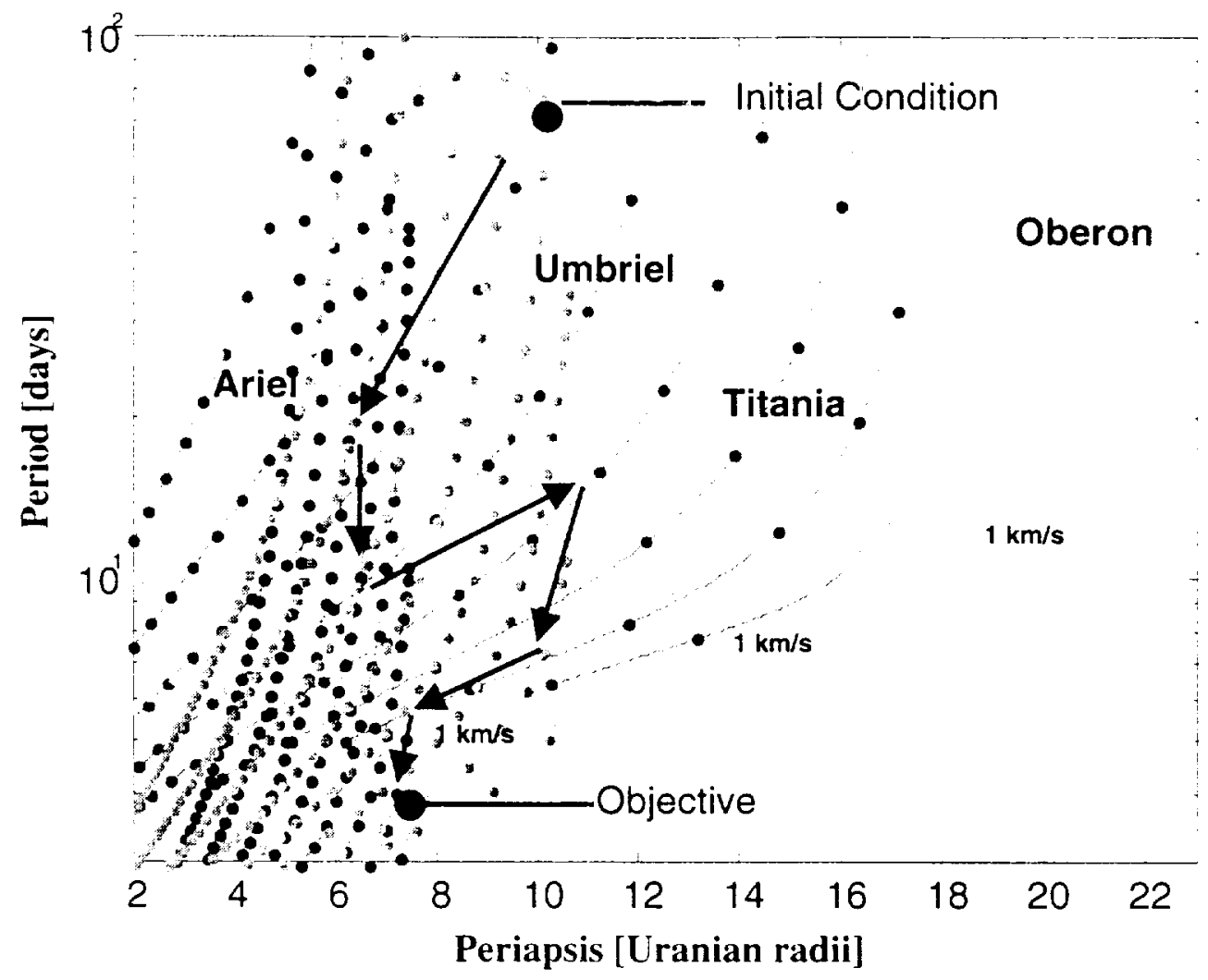

Figure 3 Uranian Tisserand graph showing the mission objective, the initial condition, and the initial strategy employed for Uranian tour design. The $V_{\infty} s$ at each satellite range in value from $1 \mathrm{~km} / \mathrm{s}$ to $8 \mathrm{~km} / \mathrm{s}$ in increments of $1 \mathrm{~km} / \mathrm{s}$. Each tick mark separates flyby altitudes of $100 \mathrm{~km}$.

Once the initial phase of the tour is complete (and the inclination is reduced) the next step is to select a path for the middle of the tour. As with the Europa Orbiter design. this is the least constrained of the three phases, because a large number of paths are possible. Tour design strategy in the middle phase consists of reducing the energy of the orbit while setting up the right conditions to begin the end phase of the tour.

The end phase of the tour is essentially determined by the orbit state of the final objective (i.e., the location of the final orbit on a Tisserand graph). The final orbit state by its very nature determines the last few flybys of the tour. For instance, in design studies, ${ }^{2}$ the Europa Orbiter tour typically ends with a transfer from Callisto to Ganymede, multiple resonant flybys of Ganymede. and then a transfer from Ganymede to Europa. We can anticipate that a similar end-of-tour strategy can be developed for our objective of achieving a low arrival $V_{\infty}$ at Ariel (less than $1 \mathrm{~km} / \mathrm{s}$ ).

The various phases of the tour design process can now be explained and a tour design strategy derived with the Uranian Tisserand graphs. Figure 4 is a Uranian Tisserand graph that includes the initial condition, the mission objective, and a general strategy of path selection for all three phases. This suggested design strategy is only a first-glance, "broad brush" assessment of what is possible. The initial phase of cranking down 
inclination is represented in rigure 4 as the first arrow rom the top. The second arrow from the top begins the middle phase of the iour, using Umbriel and Ariel to pump down the energy of the orbit while maintaining a relatively high periapsis. The next three arrows in the progression indicate the strategy for the rest of the middle phase. and are not intended to represent the actual path selection (since many paths are possible in the middle phase). Rather, those three arrows show how the tour must move to the right on the Tisserand graph in order to reach the mission objective of $\mathrm{V}_{\infty}<1 \mathrm{~km} / \mathrm{s}$ at Ariel. In general, in the middle phase of the tour design, Oberon is used to pump up, while Titania and Umbriel are used to pump down. The pump up is necessary in order to be able to reach the $1 \mathrm{~km} / \mathrm{s}$ mission objective. (The most efficient way to accomplish that is to approach Ariel from the right side of the Tisserand graph, hence we use Oberon for pumping up.)

The end phase for the tour at Uranus is more flexible than the end phase for the Europa Orbiter. The reason for this is that the lack of a radiation constraint at Uranus allows the end phase flybys to use Umbriel (which is closer to Uranus than Titania) extensively, while at Jupiter the need to keep the periapsis as high as possible limited the use of multiple Ganymede-Europa transfers for the final approach to Europa. So, for the end phase of our tour design at Uranus, the final approach can use Titania, Umbriel or a combination thereof. (Oberon is not an option, because no orbit from Oberon can reach Ariel with an arrival $\mathrm{V}_{x}<$ $1 \mathrm{~km} / \mathrm{s}$.) The theoretical best arrival $V_{\infty}$ at Ariel is $0.46 \mathrm{~km} / \mathrm{s}$, via a Hohmann transfer from Umbriel. while the Hohmann transfer from Titania results in a $V_{\infty}$ of arrival at Ariel of 1.00 $\mathrm{km} / \mathrm{s}$. The tour that appears in Table 5 was designed using the strategy just described. This tour is designated "U00-0l", for the first Uranian tour designed in 2000. Tour U00-01 uses nine flybys and requires 261 days to reduce the initial inclination of 13.6 degrees. While nine flybys are more than required for inclination reduction in any Europa Orbiter tour, nine is not an unreasonable number. The tour certainly demonstrates that such a large inclination can be handled.

Events 11 through 32 in Table 5 represent the middle phase of the tour. The middle phase makes extensive use of Ariel itself for pump downs, and includes several multiple flybys of Ariel. Many of these flybys are non-resonant. Resonant flybys are more difficult to achieve for Uranian satellites than Jovian satellites, due to the weaker gravity of the Uranian satellites (i.e.. the tick marks on the Tisserand graph are closer together). Therefore, in Tour L00-01, non-resonant "repeat" flybys are used at every satellite with the exception of Oberon. The middle phase achieves its goal of setting up the tour for the end phase, but by no means is the middle phase optimized for number of flybys or the best path selected. since Tour LOO-01 is intended only as a demonstration of potential.

The end phase of U00-01 occurs between events 32 and 40 and consists of multiple flybys of Titania followed by multiple flybys of Umbriel with a final transfer orbit from Umbriel to Ariel. A close inspection of Figure 4 reveals that this is the natural path to follow from energy considerations. Again, many of the multiple flybys of Titania and Umbriel in the end phatse are non-resonant. The end phase achieves a $V_{\infty}$ of $0.92 \mathrm{~km} / \mathrm{s}$ at Ariel (which is sufficiently below the $1 \mathrm{~km} / \mathrm{s}$ goal). 
Table 5

TOUR U00-01 SUMMARY

\begin{tabular}{|c|c|c|c|c|c|c|}
\hline Event \#/Satellite & $\frac{\text { Altitude }}{(\mathrm{km})}$ & $\frac{\theta^{a}}{\text { (deg) }}$ & $\underline{v}_{(\mathrm{km} / \mathrm{s})}$ & $\frac{\text { Period }}{\text { (days) }}$ & $\frac{\text { Periansis }}{\left(R_{C}\right)}$ & $\frac{\text { Time }}{\text { (days) }}$ \\
\hline 1/Titania & 316 & .22 & 3.27 & 52.2 & 9.1 & 0 \\
\hline 2/Titania & 74 & -65 & 3.27 & 43.5 & 8.6 & 52.2 \\
\hline 3/Titania & 58 & -54 & 3.27 & 34.8 & 8.1 & 95.8 \\
\hline 4/Titania & 54 & -23 & 3.27 & 26.1 & 7.4 & 130.6 \\
\hline 5/Titania & 90 & -92 & 3.28 & 26.1 & 7.2 & 156.7 \\
\hline 6/Titania & 90 & -92 & 3.28 & 26.1 & 7.0 & 182.8 \\
\hline 7/Titania & 90 & -91 & 3.29 & 26.1 & 6.9 & 208.9 \\
\hline $8 /$ Titania & 90 & -91 & 3.30 & 26.1 & 6.8 & 235.1 \\
\hline 9/Titania & 776 & -103 & 3.30 & 27.0 & 6.9 & 261.2 \\
\hline 10/Oberon & +14 & 0 & 2.98 & 23.2 & 6.3 & 287.6 \\
\hline 11/Ariel & 378 & 0 & 3.04 & 20.2 & 6.2 & 312.2 \\
\hline 12/Ariel & 55 & 0 & 3.04 & 16.7 & 6.1 & 332.4 \\
\hline 13/Titania & 388 & 0 & 3.18 & 14.5 & 5.7 & 348.2 \\
\hline 14/Umbriel & $5+$ & 180 & 3.64 & 13.2 & 5.5 & 364.3 \\
\hline 15/Oberon & 584 & 0 & 2.60 & 14.5 & 6.1 & 378.7 \\
\hline 16/Ariel & 1.33 & 180 & 2.99 & 12.6 & 6.0 & 406.2 \\
\hline 17/Ariel & 219 & 180 & 2.99 & 11.2 & 6.0 & 418.8 \\
\hline 18/Ariel & 119 & 0 & 2.99 & 9.9 & 5.9 & 440.7 \\
\hline 19/Oberon & 109 & 180 & 2.13 & 11.5 & 7.1 & 448.9 \\
\hline 20/Ariel & 651 & 0 & 2.00 & 8.8 & 7.0 & 473.6 \\
\hline 21/Ariel & 88 & 180 & 1.87 & 10.2 & 7.1 & 491.3 \\
\hline 22/Ariel & 251 & 0 & 1.86 & 11.4 & 7.2 & 501.8 \\
\hline 23/Oberon & 282 & -12 & 2.10 & 12.5 & 9.0 & 526.5 \\
\hline 24/Umbriel & +32 & -101 & 1.97 & 12.8 & 9.0 & 561.6 \\
\hline 25/Umbriel & 196 & 180 & 1.97 & 11.3 & 8.9 & 575.2 \\
\hline 26/Oberon & 4() 4 & 180 & 1.78 & 1.3 .2 & 10.3 & 583.8 \\
\hline 27/Umbriel & 60 & 0 & 1.20 & 11.8 & 10.2 & 599.2 \\
\hline $28 /$ Titania & 286 & 0 & 1.93 & 9.7 & 9.0 & 609.8 \\
\hline 29/Oberon & $2+1$ & 180 & 1.44 & 11.7 & 11.2 & 6.37 .7 \\
\hline 30/Titania & 342 & 0 & 1.72 & 9.5 & 9.9 & 662.1 \\
\hline 31/Umbriel & 166 & 180 & 1.29 & 8.3 & 9.7 & 682.8 \\
\hline 32/Umbriel & 151 & 180 & 1.29 & 7.3 & 9.5 & 691.1 \\
\hline 33/Titania & 909 & 138 & 1.30 & 9.9 & 1.3 .3 & 696.1 \\
\hline $34 /$ Titania & 1293 & 139 & 1.04 & 8.7 & 12.2 & 709.2 \\
\hline $35 /$ Titania & 95 & -180 & 1.04 & 6.5 & 9.3 & 717.9 \\
\hline $36 /$ Titania & 2189 & -173 & 1.04 & 6.1 & 8.4 & 744.0 \\
\hline 37/Umbriel & 238 & -180 & 1.61 & 5.5 & 8.1 & 760.7 \\
\hline 38/Umbriel & 77 & -180 & 1.61 & 5.0 & 7.7 & 777.2 \\
\hline 39/Umbriel & 519 & -180 & 1.61 & 4.7 & 7.5 & 802.1 \\
\hline 40/Ariel & 316 & & 0.92 & & & 810.8 \\
\hline
\end{tabular}

${ }^{\mathrm{d}} \theta$ is the angle in the plane perpendicular to the incoming $V_{\infty}$ vector; where values of 0 and 180 deg correspond (approximately) to equatorial thybys, and -90 and +90 deg correspond (approximately) to Hybys over the north and south poles of the satellite, respectively. 


\section{CONCLUSIONS}

In this paper, we demonstrate the feasibility of a Galileo-style tour of the Uranian satellites. We use the Tisserand graph method (originally derived for Europa Orbiter tour design), thereby demonstrating the power of this graphical technique. We derive various practical guidelines and constraints for tour design at Uranus and show that low arrival $\mathrm{V}_{x}$ at Ariel can be achieved within 2.5 years. Thus, a tour of the Uranian satellites is both feasible and practical.

\section{REFERENCES}

1. Heaton, A. F., "A Systematic Method for Gravity-Assist Tour Design", Master's Thesis, Purdue University, West Lafayette, IN, 47901, December 2000.

2. Heaton, A. F., Strange, N. J., Longuski, J. M., and Bonfiglio, E., "Automated Design of the Europa Orbiter Tour", AIAA Paper No. 2000-37, AAS/AIAA Astrodynamics Specialist Conference, Denver, Colorado, August 2000. Also, to appear in the Journal of Spacecraft and Rockets.

3. Strange. N. J., and Longuski, J. M., “A Graphical Method of Gravity-Assist Tour Design", AIAA Paper No. 2000-4030. AAS/AIAA Astrodynamics Specialist Conference, Denver, Colorado, August 2000. Also, to appear in the Journal of Spacecraft and Rockets.

4. Johnson, Wyatt, and Longuski, James M., "Design of Aerogravity Assist Trajectories", AIAA Paper No. 2000-4031. Denver, Colorado, August 2000. Also, to appear in the Journal of Spacecruft and Rockets.

5. Labunsky. A.V., Papkov, O.V., Sukhanov, K.G., Multiple Gravity Assist Interplanetary Trajectories. Overseas Publishers Association, 1998. Amsterdam, The Netherlands.

6. Roy, A. E., Orbital Motion, Adam Hilger Co., 1982, pp. 129-130.

7. Rinderle, E. A., "Galileo User's Guide, Mission Design System. Satellite Tour Analysis and Design Subsystem," Jet Propulsion Laboratory, California Institute of Technology, Pasadena, CA. JPL Internal Document D-263, July 1986.

8. Williams, S. N., "Automated Design of Multiple Encounter Gravity-Assist Trajectories," Master's Thesis. School of Aeronautics and Astronautics, Purdue Liniversity. August 1990. 
9. Longuski, J. M. and Williams, S. N., "Automated Design of Gravity-Assist Trajectories to Mars and the Outer Planets," Celestial Mechanics and Dynamical Astronomy. Vol. 52. No. 3. 1991, pp. 207-220.

10. Patel, M. R., "Automated Design of Delta-V Gravity-Assist Trajectories for Solar System Exploration," Master's Thesis, School of Aeronautics and Astronautics, Purdue University, August 1993.

11. Bonfiglio, E. P., "Automated Design of Gravity-Assist and Aerogravity-Assist Trajectories" Master's Thesis, School of Aeronautics and Astronautics, Purdue University, August 1999.

12. Johanessen, Jennie R., "Europa Orbiter Mission Design Trades: Summary of Trajectory Results to Date." Presentation, Jet Propulsion Laboratory, California Institute of Technology, Pasadena, California, October 4, 1999. 